\title{
Incidence of Plate Removal in Maxillofacial Region: A Single Centred Retrospective Study
}

\author{
Rezin Ahmed ${ }^{1}$, M.R. Muthusekhar ${ }^{2}$ and Pradeep D ${ }^{3}$ \\ ${ }^{1}$ Saveetha Dental College \& Hospitals Saveetha Institute of Medical and Technical Sciences, \\ Saveetha University,Chennai 600077 Tamilnadu, India. \\ ${ }^{2,3}$ Department of Maxillofacial surgery Saveetha Dental college \& Hospitals, Saveetha Institute \\ of Medical and Technical Sciences, Saveetha University,Chennai 600077 Tamilnadu, India. \\ Corresponding author email: pradeep@saveetha.com
}

\begin{abstract}
The long-term management of miniplate fixation osteosynthesis remains debatable and controversial with few authors advocating routine removal of the miniplates after 3-6 months of placement, while others recommend retention of the miniplates unless their removal is clinically indicated. The aim was to study the incidence, indications, time gap, role of metallic composition and site of removal of miniplates in operated cases of maxillofacial region over a one-year period. Patients undergoing removal of miniplates over a one-year period were studied and evaluated regarding the number of miniplates removed, time gap present between fixation and removal of miniplates, indications for removal, metallic composition of miniplates removed, sites of removal and complications. Correlations between indications for miniplate removal based upon time gap, metallic composition, age of patients undergoing plate removal and number of miniplates present were determined using Chi-square test. Correlation between metallic composition of miniplate and time gap was also determined using Chi-square test. The miniplates were removed in 31 patients (26 males and 5 females). Most common indication for removal was infection (45\%). Forty-five percent of the patients underwent miniplate removal within 1 year of placement. The correlation between indications for miniplate removal and time gap was found to be statistically significant $(\mathrm{P}=0.04)$. Most of the hardware removal is performed subsequent to complications associated with hardware and local factors. There is no significant association between the composition of the hardware and pate removal. However there is a significant association between the time gap and indication for plate.Miniplate removal should be performed when hardware is causing various complications and physical symptoms. Infection, miniplate exposure, pain, palpability or any other morbidity that appears after bony union should be treated by miniplate removal
\end{abstract}

KEY WORDS: PLATE FIXATION, FRACTURE, INFECTION, MINIPLATE, TRAUMA.

\section{INTRODUCTION}

In the era of increasing auto mobilization, industrialization and technology, the treatment of maxillofacial injuries has attained a prominent position. Road traffic accidents, which are becoming more and more frequent, particularly have brought about an increase in maxillofacial injuries. The highest number of trauma occurred in the age group of

Biosc Biotech Res Comm P-ISSN: 0974-6455 E-ISSN: 2321-4007

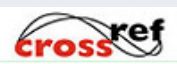

Identifiers and Pagination

Year: 2021 Vol: 14 No (10) Special Issue

Pages: 109-114

This is an open access article under Creative

Commons License Attribn 4.0 Intl (CC-BY).

DOI: $h t t p: / / d x$.doi.org/10.21786/bbrc/14.10.18
20-29 years constituting $44.5 \%$ of all trauma cases seen over a 9-year period. The male-to-female ratio in this study was found to be 6.2:1, which is lower compared to other studies. The other causes of maxillofacial injuries are interpersonal violence, falls, sporting injury and industrial trauma the most common bone involved was the mandible (64.4\%), and the most common site in the mandible was the parasymphysis (25.3\%), followed by the angle (16.2\%). Champys described the ideal lines of the osteosynthesis on which plates have to be applied to miniplates are small size and easily adapted monocortically on bone (Abhinav et al., 2019).

They provide functional stability since the system is biomechanically balanced. But one of the most significant drawbacks was the phenomenon of stress shielding atrophy of the bone under the rigid plate which makes the bone vulnerable to refracture once the plates were removed.
Article Information

Received: $09^{\text {th }}$ Aug 2021

Accepted after revision: $15^{\text {th }}$ Oct 2021 
Although gold, silver, copper and its alloys lead and aluminium and its alloys were tested. Stainless steel emerged through the era as the new corrosion resistant material. At about the same time or later on the other metals or alloy like titanium were introduced with claims of advantages over the classic stainless steel. Stainless steel and titanium plates are also being used in lefort osteotomies. The management of disposal of bio wastes also has to be done to prevent iatrogenic injuries (Christabel et al., 2016; Kumar and Rahman, 2017; Jain et al., 2019).

The most commonly reported indications for maxillofacial hardware removal include infection at the site of surgery and/or hardware extrusion or exposure. Murthy and Lehman reported that most infections after fixation surgery for maxillofacial trauma occur in the mandible and are the major cause of miniplate removal. Studies have reported various values for the removal rate of miniplates, ranging from $7 \%$ to $33.8 \%$. Some researchers recommend removal in general, while others do not recommend removal unless clinical symptoms or complications occur. Clear evidence for such a recommendation has not yet been established (Murthy and Lehman, 2005).

This study analysed the incidence, indications, time gap, metallic composition of miniplates removed and site of removal of miniplates in one year study period in operated cases of maxillofacial region .Previously our team had conducted numerous clinical trials (Jesudasan et al., 2015; Christabel et al., 2016; Mp, 2017a; Mp and Rahman, 2017; Packiri et al., 2017; Patil et al., 2017; Marimuthu et al., 2018) and lab animal studies (Kumar and Sneha, 2016; Kumar, 2017; Mp, 2017b; Rao and Kumar, 2018; Abhinav et al., 2019) and in-vitro studies (Patturaja and Pradeep, 2016; Abhinav et al., 2019) over the past 5 years. Now we are focussing on epidemiological surveys and retrospective studies. The idea for this retrospective study stemmed from the current interest in our community.

\section{MATERIAL AND METHODS}

Sample size: This retrospective study was conducted in the university setting. Data chosen for evaluation were patients who reported to a private dental college for the removal of plates fixed in maxillofacial region. The details of the patients were obtained from analysis of 86,000 patients from June 2019 to March 2020 from patient dental records. The study was conducted after getting ethical approval from the Institutional Ethical Committee (Ethical Approval Number: SDC/SIHEC/2020/DIASDATA/0619-0320). Cross verification was done with the help of patient dental records data. To minimise sampling bias all data were included.

Study design: Data collected comprised age, gender, reason for removal of miniplates, site of removal, length of time between surgery and removal of the miniplate, number of miniplates removed, metallic composition of miniplates and intra-operative and post-operative complications following miniplate removal. The reasons for removal were classified into the following categories: patient's request for removal; infection; pain without signs of infection; asymptomatic miniplate exposure; pediatric trauma; prosthetic rehabilitation; and others.

Statistics: The site of miniplate removal included mandible and midface. Correlations between indications for miniplate removal based upon time gap, metallic composition, age group and number of miniplates present were determined using Chi-square test. Correlation between metallic composition of miniplate and time gap for removal was also determined using Chi-square test.

\section{RESULTS AND DISCUSSION}

31 patients underwent miniplate removal. There were 26 males $(80 \%)$ and 5 females $(20 \%)$, with an average age of approximately 32.5 years (range, 4 - 65 years). Miniplates were removed in 16 cases $(80 \%)$ from mandible and four cases $(20 \%)$ from the midface region. In the mandible (16 cases), body and symphysis region were most commonly involved ( 5 cases each $-31.25 \%$ ) followed by angle region $(18.75 \%)$, and condyle (6.25\%). However, multiple sites were involved in only 2 cases $(12.5 \%)$.

Out of miniplates removed, stainless steel and titanium material shared an equal percentage (17 miniplates in 10 patients in each category). Out of 118 screws, 62 (52.54\%) were stainless steel and $56(47.46 \%)$ were titanium. There were 9 cases (45\%) in which the miniplates were removed due to infection and in 6 cases (30\%), miniplates were removed due to complaint of pain without any sign of infection. Prosthetic rehabilitation, asymptomatic miniplate exposure and patient request needed miniplate removal in 1 case each (5\%). One patient was a 4 years old child and, in another patient, malunion subsequent to inadequate reduction led to deranged occlusion requiring miniplate removal.

Figure 1: This pie chart represents the different sites from which the miniplates were removed. The blue colour represents Angle of the mandible from which $13.33 \%$ of plates were removed. The green colour represents Body of the mandible with $33.33 \%$, Grey colour represents condyle with $\mathbf{6 . 6 7 \%}$, Yellow represents the symphysis with $33.33 \%$ and violet represents Multiple sites with $13.3 \%$.

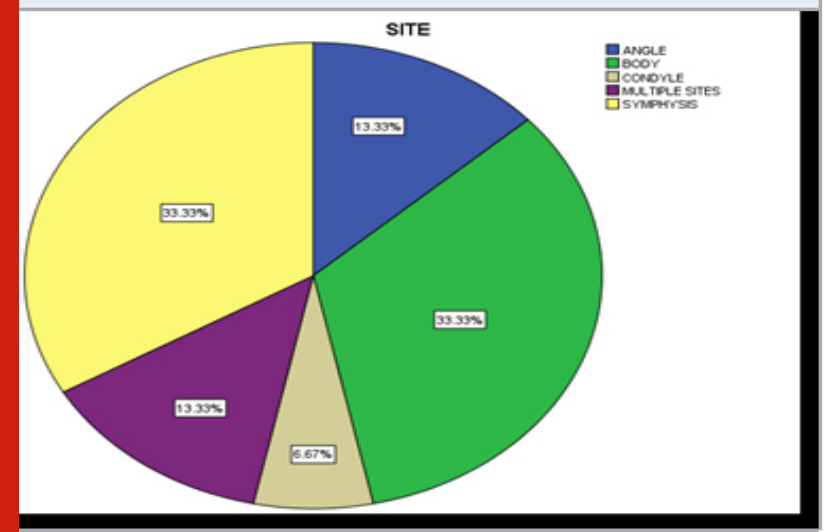

Three cases (15\%) each were performed within 3 months, within 3 to 6 months and within 6 to 12 months, 5 cases $(25 \%)$ within $1-2$ years and 6 cases $(30 \%)$ in which removal 
was performed after more than 2 years of first surgery. There was a higher incidence of miniplate removal (9 cases $45 \%$ ) within one year of first surgery. Removal of miniplate was performed within 3 months in three cases because of infection involving bone, pediatric care which requires removal of the hardware as it can hinder the growth of the bone, and malunion subsequent to inadequate reduction in one case each, respectively. One case was an operated case of orthognathic surgery in which miniplate from zygomatic buttress was removed due to pain subsequent to miniplate exposure at the site. Among these 20 cases, there were 9 cases in which miniplate fixation was done at other sites also but were not indicated for removal.

Figure 2: This pie diagram represents indication for the plate removal. Blue represents infection, green represents pain, grey represents patients demand and violet indicates pediatric trauma.55.56 \% of cases are due to infection, $33.3 \%$ due to pain.

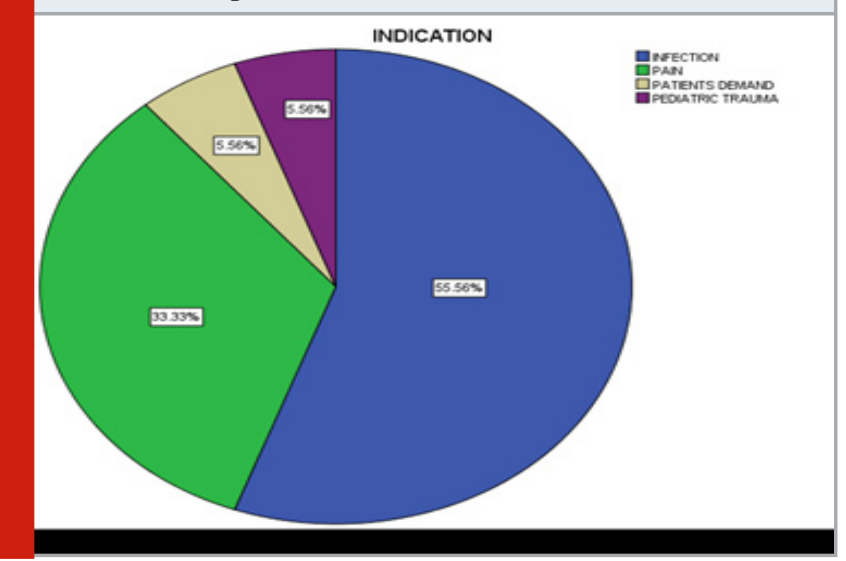

Figure 3: This chart represents the association between the type of material and the indication for plate removal. $\mathrm{X}$ - axis represents the Reason for plate removal and $\mathrm{Y}$ - axis represents the frequencies of plate removal in the different type of material used. Chi square was done and association was found to be statistically not significant; $p$ value:0.85 ( $>0.05$ ) proving that there was no association between indication for plate removal and type of plate material

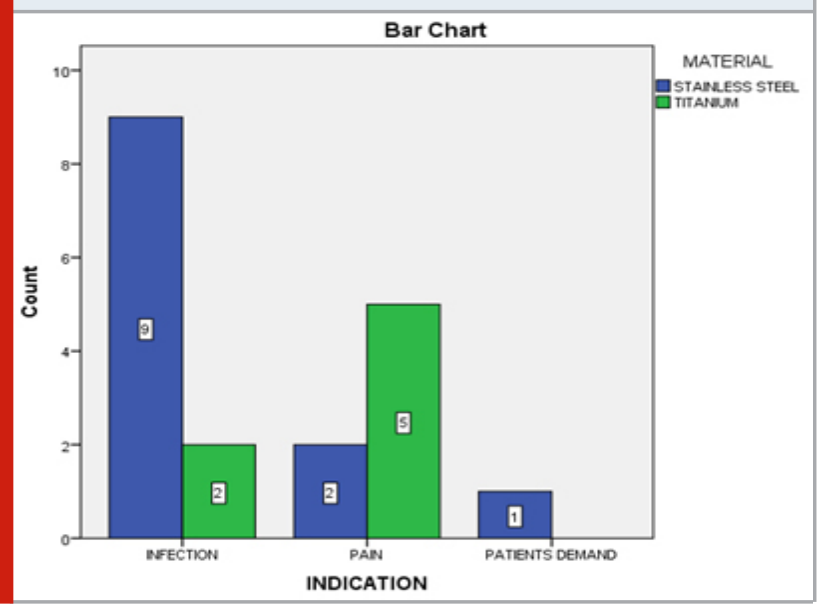

Several metals have been used since the 1920's for manufacturing hardware for fixation of maxillofacial trauma. Although gold, silver, copper, lead and aluminium were tested, stainless steel emerged through the era as the corrosion resistant material. Later on, at about the same time, titanium gained popularity with advantages over the traditional stainless steel. Titanium was first reportedly used around 1940's and was not only biocompatible metal, it also had a tendency for osseointegration and had excellent corrosion resistance. It also had excellent ductility and tensile strength and was totally non-toxic. Removal of miniplates has remained controversial. According to researchers, who oppose removal of an asymptomatic miniplate, biocompatibility of material, low incidence of complications, the risks of general anesthesia during removal, possible damage to adjacent anatomical structures and the expense of removal contraindicate removal of asymptomatic miniplate. On the contrary, authors who favor removal argue that the miniplate can possibly act as a foreign object with the potential to cause complications, and also miniplates generate growth restrictions among pediatric patients (Deepak et al., 2011; Park et al., 2016; Kumar and Rahman, 2017; Jain et al., 2019).

Champy recommended routine removal of all miniplates after 3 months of fixation and this concept became standard. Later, Vitallium gained acceptance as a more inert implant material and authors advocated retention of vitallium miniplates. Frost et al. (1983) studied the fate of vitallium miniplates and reported $18 \%$ removal rate on clinical grounds. Around the same time, it was shown that titanium (Ti) has startling success in many surgical procedures. According to Meningaud et al., almost $100 \%$ of $\mathrm{Ti}$ is released at local sites during the osteosynthesis, however, Ti levels remain constant and stable in the surrounding tissues and remain clinically inert. Removal of Ti miniplates was not accepted as routine procedure except in the case of infection, dehiscence, hypersensitivity or screw loosening (Michelet et al., 1973; Frost, El-Attar and Moos, 1983; Brown et al., 1989; Meningaud et al., 2001; Rao and Kumar, 2018; Abhinav et al., 2019).

Matthew et al. concluded that removal of miniplates and screws should be performed mainly to treat symptoms caused by the implants. advocated routine removal of stainless steel miniplates after 3 months to prevent interference with jaw function, as miniplates prevent transmission of functional stress to the site, subsequently leading to osteoporosis and weakening of bone. also recommended routine removal of miniplates due to stress shielding effect. In a retrospective study of 279 Champy stainless steel miniplates fixed as permanent implants, Brown et al. challenged this practice of routine removal of stainless steel miniplates 3 or 4 months after insertion. The main reason for the removal of the miniplate in our study was infection at the surgical site. In literature also, the most common indication reported for miniplate removal is infection involving the site. However patient demand is the most common indication as cited in a study (Kennady et al., 1989; Meningaud et al., 2001; Park et al., 2016; Kumar and Rahman, 2017; Jain et al., 2019). 
Miniplates are often located in thin submucosa, which results in exposure to traumatic environmental effects. The masticatory forces acting on the miniplates or screws may compromise interfragmentary stability and consequently, screws may loosen resulting in inflammation which increases the possibility of infection. Poor suturing techniques and inadequate bone cooling during the screw hole preparation have also been suggested as causes of miniplate failure due to infection. Patients receiving injuries in road traffic accidents often have contaminated wounds which increases the incidence for miniplate removal in future. The infective course associated with miniplates is normally a well localised reaction within the bone and does not develop osteomyelitis or delayed union. Within the first 6 weeks after fixation, the infection can be managed conservatively by draining the pus out and antibiotic therapy (local as well as systemic) (Islamoglu et al., 2002; Rao and Kumar, 2018; Abhinav et al., 2019).

Table 1. Pearson correlation between parameters (variables).

\begin{tabular}{|l|c|c|c|}
\hline S. No. & Variables & $\begin{array}{c}\text { Pearson Chi } \\
\text { Square value }\end{array}$ & Sig. \\
\hline a. & Time gap and indication for removal & 9.69 & $0.04^{*}$ \\
\hline b. & Metallic composition and indication for removal & 0.31 & 0.85 \\
\hline c. & Age of patient and indication for removal & 4.48 & 0.61 \\
\hline d. & $\begin{array}{c}\text { Number of hardware present } \\
\text { and indication for removal }\end{array}$ & 3.06 & 0.80 \\
\hline e. & Metallic composition and time gap & 0.31 & 0.85 \\
\hline
\end{tabular}

This permits fracture to heal while the bone remains splinted and fixed. Once the fracture is clinically stable and healed, the miniplate may then be removed. If the infection does not involve underlying operated bone, the existing miniplates can generally be preserved by antibiotics, irrigation, debridement and removal of the nidus, such as a necrotic tooth or soft tissue. However, if infection involves bone and bony union has also not occurred, miniplate removal is indicated which may be followed by external fixation and bone grafting. Tooth damage during fixation surgery or involvement of tooth or teeth in the line of fracture at time of trauma led to subsequent development of infection in 4 out of 9 infection-related cases. In 4 infection cases, screw loosening led to infection. Impaired healing due to compromised blood supply. 14\% incidence of pain after surgery at or around the site of fixation, whereas reported pain in 24\% cases (Bhatt et al., 2005; Rosa et al., 2016; Rao and Kumar, 2018; Abhinav et al., 2019).

In our study, pain was reported to be the cause of miniplate removal in 6 patients $(30 \%)$. Pain was subsequent to nerve compression by miniplate in two cases; tooth damage, miniplate palpability, malunited condylar fracture and miniplate exposure in one case each. Other symptoms or conditions contributing for miniplate removal include patient request, pediatric growth restriction, prosthetic rehabilitation, miniplate exposure, deformities due to malunion or non-union, tooth extraction, screw loosening, wound dehiscence, palpability, nerve damage, cold intolerance, etc. In our study, only one patient requested asymptomatic miniplate removal and rest of the hardware removal were performed subsequent to associated hardware complications.

In pediatric patients, miniplates should be removed within two to three months after fracture surgery due to the potential of growth restriction. In a four-year retrospective study 912 pediatric patients underwent treatment by conventional methods using metal fixation for maxillofacial fractures. All patients underwent a second surgery to remove the nonresorbable materials 6 - 8 months after the first surgery to prevent long-term growth disturbance. With regard to the length of time from first surgery to miniplate removal, most cases (45\%) involved removal within one year. Majority of the miniplate removal, as reported in the literature, occurred within 6 months to 1 year of fixation. A few authors even reported miniplate removal at less than 3 months of fixation. Routine removal of the miniplates should be performed after confirmation of bone healing and principally should be performed between 6 months and a year (Haug et al., 2003; Yamamoto et al., 2015; Conti et al., 2016; Rao and Kumar, 2018; Abhinav et al., 2019).

Most of the miniplates $(80 \%)$ were removed from mandible. Removal of the miniplate from the mandible was most often performed at the mandibular body and symphysis region. concluded that mandibular angle region is most common site for miniplate removal (39.5\%). Author reported $62.85 \%$ incidence of involvement of mandible for hardware removal in maxillofacial region. conducted low-vacuum scanning electron microscopy (SEM) and concluded that no distinguishable difference exists in the surface characteristics of either stainless steel or titanium miniplates removed at 4,12 and 24 weeks after surgery. Energy-dispersive X-ray (EDX) identified aluminium and silicon deposits over the flat surfaces of these miniplates. Hence, it was not evident to support the routine removal of either titanium or stainless steel miniplates subsequent to surface corrosion up to 6 months after implantation (Matthew et al., 1996; Islamoglu et al., 2002; Yamamoto et al., 2015; Park et al., 2016).

In the biologic environment, stainless steel degrades by combination of electrochemical corrosion and wear and titanium degrades mainly due to wear and particle release. Corrosion and wear products (metal ions or particles) may 
lead to changes in the surrounding tissues, ranging from fibrosis to infection and necrosis. Because of associated complications like corrosion, toxicity, hypersensitivity and stress protection, stainless steel should not be considered as a permanent fixation device in maxillofacial region. However, due to the absence of any untoward reaction of bone and soft tissues, superior corrosion resistance, noncarcinogenicity, hyposensitivity, nontoxicity and excellent tissue compatibility, the removal of titanium hardware, subsequent to their fixation, can be harmlessly avoided and can be retained as permanent implants in maxillofacial region also reported osteolysis and necrosis around stainless steel implants due to electrolysis.

However, we found that the role of metallic composition (either stainless steel or titanium) is negligible as the number of miniplates removed were equal for stainless steel and titanium metal. Intraoperatively, we encountered osseointegration of screws in three cases wherein the metallic composition of hardware was titanium. Linder and Lundskog found that the bone formed around the titanium screws was dense, which might cause difficulty in retrieving the titanium screws being firmly adherent. We found co-relations between indications for miniplate removal based upon time gap, metallic composition, age group and number of miniplates present; and correlation between metallic composition of miniplate and time gap for removal was also determined using Chisquare test ( Venable et al., 1937; Linder and Lundskog, 1975; Torgersen and Gjerdet, 1994; Haug, 1996; Rao and Kumar, 2018; Abhinav et al., 2019).

The association between indications for miniplate removal and time gap was found to be statistically not significant. Chi square value was 0.31 and the significance was $0.85(>0.05$. time gap had a major role in development of specific symptoms, it was found to be statistically significant. Infection was the common indication for miniplate removal within a time gap of 1 - 2 years. After two years of miniplate fixation, pain and infection led to miniplate removal. Within one year of miniplate placement, other factors were prevalent which led to miniplate removal. However, association between indications for miniplate removal and metallic composition; age group; and number of miniplates present were non-significant. Metallic composition of miniplate, age of patient and number of hardware fixed in first surgery had no role in development of causative factors like pain, infection, etc. Correlation between metallic composition of miniplate and time gap for removal was also determined using Chi-square test and found to be non-significant.

\section{CONCLUSION}

Most of the hardware removal is performed subsequent to complications associated with hardware and local factors. There is no significant association between the composition of the hardware and pate removal. However, there is a significant association between the time gap and indication for plate. Miniplate removal should be performed when hardware is causing various complications and physical symptoms. Infection, miniplate exposure, pain, palpability or any other morbidity that appears after bony union should be treated by miniplate removals.

\section{ACKNOWLEDGEMENTS}

The authors would like to acknowledge the chancellor, Director of academics ; the Principal, and the Vice Chancellor, Saveetha University; Associate dean of research , HOD and their professors, readers, lecturers, and their fellow postgraduates, Department of Oral and Maxillofacial Surgery, Department of periodontics, Saveetha University, the support from their parents, and from their family

\section{REFERENCES}

Abhinav, R. P. et al. (2019). The Patterns and Etiology of Maxillofacial Trauma in South India. Annals of maxillofacial surgery, 9(1), pp. 114-117.

Abhinav, R. P., Sweta, V. R. and Ramesh, A. (2019). Role of virtual reality in pain perception of patients following the administration of local anesthesia. Annals of Maxillofacial Surgery, p. 110. doi: 10.4103/ams.ams_263_18.

Bhatt, V., Chhabra, P. and Dover, M. S. (2005). Removal of Miniplates in Maxillofacial Surgery: A Follow-Up Study. Journal of Oral and Maxillofacial Surgery, pp. 756-760. doi: 10.1016/j.joms.2005.02.005.

Brown, J. S. et al. (1989). The fate of miniplates in facial trauma and orthognathic surgery: A retrospective study. British Journal of Oral and Maxillofacial Surgery, pp. 306-315. doi: 10.1016/0266-4356(89)90043-0.

Christabel, A. et al. (2016). Comparison of pterygomaxillary dysjunction with tuberosity separation in isolated Le Fort I osteotomies: a prospective, multi-centre, triple-blind, randomized controlled trial. International Journal of Oral and Maxillofacial Surgery, pp. 180-185. doi: 10.1016/j. ijom.2015.07.021.

Conti, G., Bardellini, E. and Amadori, F. (2016). Dental radiography: Embarrassing foreign objects. British Dental Journal, pp. 3-3. doi: 10.1038/sj.bdj.2016.471.

Deepak, S., Manjula, S. and Others (2011). Comparison of Titanium bone plates and screws vs. stainless steel bone plates and screws in the management of mandibular fractures--A long term clinical study. International Journal of Clinical Dental Science, 2(3). Available at: http://edentj. com/index.php/ijcds/article/view/475.

Frost, D. E., El-Attar, A. and Moos, K. F. (1983). Evaluation of metacarpal bone plates in the mandibular fracture. British Journal of Oral Surgery, pp. 214-221. doi: 10.1016/0007-117x(83)90045-8.

Haug, R. H. (1996). Retention of asymptomatic bone plates used for orthognathic surgery and facial fractures. Journal of Oral and Maxillofacial Surgery, pp. 611-617. doi: 10.1016/s0278-2391(96)90644-8.

Haug, R. H., Cunningham, L. L. and Todd Brandt, M. (2003). Plates, Screws, and Children: Their Relationship in Craniomaxillofacial Trauma. Journal of Long-Term Effects of Medical Implants, p. 18. doi: 10.1615/ jlongtermeffmedimplants.v13.i4.20.

Islamoglu, K. et al. (2002). Complications and Removal 
Rates of Miniplates and Screws Used for Maxillofacial Fractures. Annals of Plastic Surgery, pp. 265-268. doi: 10.1097/00000637-200203000-00006.

Jain, S. V. et al. (2019). Evaluation of Three-Dimensional Changes in Pharyngeal Airway Following Isolated Lefort One Osteotomy for the Correction of Vertical Maxillary Excess: A Prospective Study. Journal of Maxillofacial and Oral Surgery, pp. 139-146. doi: 10.1007/s12663-0181113-4.

Jesudasan, J. S., Abdul Wahab, P. U. and Muthu Sekhar, M. R. (2015). Effectiveness of $0.2 \%$ chlorhexidine gel and a eugenol-based paste on postoperative alveolar osteitis in patients having third molars extracted: a randomised controlled clinical trial. British Journal of Oral and Maxillofacial Surgery, pp. 826-830. doi: 10.1016/j. bjoms.2015.06.022.

Kennady, M. C. et al. (1989). Stress shielding effect of rigid internal fixation plates on mandibular bone grafts. A photon absorption densitometry and quantitative computerized tomographic evaluation. International Journal of Oral and Maxillofacial Surgery, pp. 307-310. doi: 10.1016/s09015027(89)80101-8.

Kumar, S. (2017). Knowledge, attitude and awareness of dental undergraduate students regarding HIV/AIDS patients. Asian Journal of Pharmaceutical and Clinical Research, p. 175. doi: 10.22159/ajpcr.2017.v10i5.17277. Kumar, S. and Rahman, R. (2017). Knowledge, awareness, and practices regarding biomedical waste management among undergraduate dental students. Asian Journal of Pharmaceutical and Clinical Research, p. 341. doi: 10.22159/ajpcr.2017.v10i8.19101.

Kumar, S. and Sneha, S. (2016). Knowledge and awareness regarding antibiotic prophylaxis for infective endocarditis among undergraduate dental students. Asian Journal of Pharmaceutical and Clinical Research, p. 154. doi: 10.22159/ajpcr.2016.v9s2.13405.

Linder, L. and Lundskog, J. (1975). Incorporation of stainless steel, titanium and Vitallium in bone. Injury, pp. 277-285. doi: 10.1016/0020-1383(75)90174-6.

Marimuthu, M. et al. (2018). Canonical Wnt pathway gene expression and their clinical correlation in oral squamous cell carcinoma. Indian journal of dental research: official publication of Indian Society for Dental Research, 29(3), pp. 291-297.

Matthew, I. R. et al. (1996). In vivo surface analysis of titanium and stainless steel miniplates and screws. International Journal of Oral and Maxillofacial Surgery, pp. 463-468. doi: 10.1016/s0901-5027(96)80085-3.

Meningaud, J.-P. et al. (2001). Dynamic study about metal release from titanium miniplates in maxillofacial surgery. International Journal of Oral and Maxillofacial Surgery, pp. 185-188. doi: 10.1054/ijom.2000.0039.

Michelet, F. X., Deymes, J. and Dessus, B. (1973). Osteosynthesis with miniaturized screwed plates in maxillo-facial surgery. Journal of maxillofacial surgery, 1(2), pp. 79-84.

Mp, S. K. (2017a). Relationship between dental anxiety and pain experience during dental extractions. Asian J Pharm Clin Res, 10(3), pp. 458-461.

Mp, S. K. (2017b). The emerging role of botulinum toxin in the treatment of orofacial disorders: Literature update. Asian J Pharm Clin Res, 10(9), pp. 21-29.

Mp, S. K. and Rahman, R. (2017). Knowledge, awareness, and practices regarding biomedical waste management among undergraduate dental students. Asian J Pharm Clin Res, 10(8), pp. 341-345.

Murthy, A. S. and Lehman, J. A., Jr (2005). Symptomatic plate removal in maxillofacial trauma: a review of 76 cases. Annals of plastic surgery, 55(6), pp. 603-607.

Packiri, S., Gurunathan, D. and Selvarasu, K. (2017). Management of Paediatric Oral Ranula: A Systematic Review. Journal of clinical and diagnostic research: JCDR, 11(9), pp. ZE06-ZE09.

Park, H.-C. et al. (2016). Mini-plate removal in maxillofacial trauma patients during a five-year retrospective study. Journal of the Korean Association of Oral and Maxillofacial Surgeons, 42(4), pp. 182-186.

Patil, S. B. et al. (2017). Comparison of Extended Nasolabial Flap Versus Buccal Fat Pad Graft in the Surgical Management of Oral Submucous Fibrosis: A Prospective Pilot Study. Journal of Maxillofacial and Oral Surgery, pp. 312-321. doi: 10.1007/s12663-016-0975-6.

Patturaja, K. and Pradeep, D. (2016). Awareness of Basic Dental Procedure among General Population. Journal of pharmacy research. indianjournals.com. Available at: http://www.indianjournals.com/ijor.aspx?target=ijor:rjpt $\&$ volume $=9 \&$ issue $=9 \&$ article $=010$.

Rao, T. D. and Santhosh Kumar, M. P. (2018). Analgesic Efficacy of Paracetamol Vs Ketorolac after Dental Extractions. Research Journal of Pharmacy and Technology, p. 3375. doi: 10.5958/0974-360x.2018.00621.2.

Rosa, J. H. et al. (2016). Review of Maxillofacial Hardware Complications and Indications for Salvage. Craniomaxillofacial Trauma \& Reconstruction, pp. 134-140. doi: 10.1055/s-0035-1570074.

Torgersen, S. and Gjerdet, N. R. (1994). Retrieval study of stainless steel and titanium miniplates and screws used in maxillofacial surgery. Journal of Materials Science: Materials in Medicine, pp. 256-262. doi: 10.1007/ bf00122394.

Venable, C. S., Stuck, W. G. and Beach, A. (1937). The effects on bone of the presence of mentals; based upon electrolysis. Annals of Surgery, pp. 917-938. doi: 10.1097/00000658-193706000-00006.

Yamamoto, K. et al. (2015). Routine removal of the plate after surgical treatment for mandibular angle fracture with a third molar in relation to the fracture line. Annals of Maxillofacial Surgery, p. 77. doi: 10.4103/22310746.161077. 